\title{
Physiologic Principles Underlying Ion Channelopathies
}

\author{
Stephen C. Cannon \\ Department of Neurology, University of Texas Southwestern Medical Center, Dallas, Texas
}

Summary: The ion channelopathies are a diverse array of human disorders caused by mutations in genes coding for ion channels. More than 40 different channelopathies have been identified, with representative disorders from every major class of ion channel and affecting all electrically excitable tissues: brain, peripheral nerve, skeletal muscle, smooth muscle, and heart. This review provides an overview of ion channel classi- fication, structure, and function as a framework for understanding which ion channel properties are altered by disease-associated mutations and how these changes disrupt cellular excitability for channelopathies affecting skeletal muscle and the CNS. Key Words: Channel mutation, electrophysiology, brain, skeletal muscle.

\section{ION CHANNELS IN HEALTH AND DISEASE}

Ion channels are macromolecular protein complexes that span the membrane lipid bilayer and facilitate the movement of ions across this hydrophobic barrier that separates the cytoplasm from the extracellular space or from intracellular organelles. (See Hille ${ }^{1}$ for a comprehensive review.) Channels create atomic-sized holes in the membrane (3-5 $\AA$ diameter), and the movement of ions occurs through these water-filled pores. Ion channels are vital to all forms of life and are highly conserved from bacteria to humans. The net movement of ions across membranes may accomplish a number of distinct biological functions. Ion flux through channels is the source of the electric current to regulate the membrane potential and thus is the fundamental basis for cellular electrical excitability. Net translocation of ions across the membrane is an important component to the regulation of osmotic balance. Flux of protons regulates $\mathrm{pH}$. Finally, ions passed through channels may be the source of a second messenger signal, such as $\mathrm{Ca}^{2+}$.

The biological role of an ion channel is determined by two functional properties. ${ }^{1}$ It is selectivity that allows one type of ion to pass more readily than others-for example. $^{2}$ The accessibility of the pore to allow ion passage as the channel shifts between closed (nonconducting) to open (conducting) conformations is a process referred to

Address correspondence and reprint requests to: Stephen Cannon, M.D., Ph.D., Department of Neurology, UT Southwestern Medical Center, 5323 Harry Hines Boulevard, Dallas, TX 55390-8813. E-mail: steve.cannon@utsouthwestern.edu. as gating, by analogy to a gate that swings open and shut over the pore. Some channels are constitutively open. The biologically rich repertoire of channel function is largely determined by the context in which the gating properties of a channel are influenced by specific stimuli (membrane voltage, ligand binding, stretch, or heat).

The activity of ion channels has been recognized as an important contributor to cellular homeostasis and maintenance of health for more than half a century. Channels have a vital role in epithelial transport (gut, kidney, lung), endocrine function (adrenals, gonads, pituitary, pancreas), contractility (heart, skeletal muscle, smooth muscle), and neuronal excitability. Modulation of channel activity by drugs is widely used for therapeutic intervention in diseases affecting these tissues. Ion channels are the primary site of action for many antiepileptic drugs, local anesthetics, migraine treatments, antipsychotics and mood stabilizers, antiarrhythmics, antihypertensives, and oral hypoglycemic agents.

A more recent concept is that ion channel dysfunction may itself be a cause of human disease. The first examples were acquired disorders caused by venoms or toxins that are potent and specific ion channel blockers (e.g., curare block of the nicotinic acetylcholine receptor) or channel modulators (e.g., $\mathrm{Na}^{+}$activation by the plant alkaloid veratridine). Next, it was recognized that the signs and symptoms for several autoimmune disorders arise from antibody binding to specific extracellular epitopes on the ion channel glycoprotein. Often these disorders occur in association with malignancy. For example, many patients with thymoma develop auto-anti- 
TABLE 1. Ion Channelopathies Affecting Brain and Skeletal Muscle

\begin{tabular}{|c|c|c|}
\hline \multicolumn{3}{|c|}{ Central Nervous System Disorders } \\
\hline Disorder & Gene & Channel Protein \\
\hline Epilepsy & $\begin{array}{l}\text { CHRNA4 } \\
\text { CHRNB2 } \\
\text { GABRA1 } \\
\text { GABRG2 } \\
\text { SCN1A } \\
\text { SCN2A } \\
\text { SCN1B } \\
\text { KCNQ2 } \\
\text { KCNQ3 } \\
\text { KCNMA1 } \\
\text { CLCN2 }\end{array}$ & $\begin{array}{l}\alpha_{4} \text { subunit, nicotinic acetylcholine receptor } \\
\beta_{2} \text { subunit, nicotinic acetylcholine receptor } \\
\alpha_{1} \text { subunit, } \mathrm{GABA}_{\mathrm{A}} \text { receptor } \\
\gamma_{2} \text { subunit, } \mathrm{GABA}_{\mathrm{A}} \text { receptor } \\
\alpha \text { subunit, } \mathrm{Na}_{\mathrm{V}} 1.1 \text { sodium channel } \\
\alpha \text { subunit, } \mathrm{Na}_{\mathrm{V}} 1.2 \text { sodium channel } \\
\beta_{1} \text { subunit, sodium channel } \\
\mathrm{KCNQ} 2 \text { potassium channel } \\
\mathrm{KCNQ} 3 \text { potassium channel } \\
\mathrm{BK} \text { potassium channel } \\
\text { ClC-2 voltage-gated chloride channel }\end{array}$ \\
\hline Familial Hemiplegic Migraine & CACNA1A & $\alpha_{1 \mathrm{~A}}$ subunit, Cav2.1 P/Q-type calcium channel \\
\hline Episodic Ataxia & $\begin{array}{l}\text { CACNA1A } \\
\text { KCN1A }\end{array}$ & $\begin{array}{l}\alpha_{1 \mathrm{~A}} \text { subunit, Cav2.1 P/Q-type calcium channel } \\
\mathrm{Kv} 1.1 \text { potassium channel }\end{array}$ \\
\hline Spinocerebellar Ataxia & $\begin{array}{l}\text { CACNAIA } \\
\text { KCNC3 }\end{array}$ & $\begin{array}{l}\alpha_{1 \mathrm{~A}} \text { subunit, Cav2.1 P/Q-type calcium channel } \\
\mathrm{Kv} 3.3 \text { potassium channel }\end{array}$ \\
\hline Hypereplexia & GLRAl & $\alpha_{1}$ subunit, glycine receptor \\
\hline \multicolumn{3}{|c|}{ Peripheral Nervous System Disorders } \\
\hline Disorder & Gene & Channel Protein \\
\hline Erythermalgia & SCN9A & $\alpha$ subunit, Nav1.7 $\mathrm{Na}$ channel \\
\hline \multicolumn{3}{|c|}{ Skeletal Muscle Disorders } \\
\hline Disorder & Gene & Channel Protein \\
\hline Periodic Paralysis & $\begin{array}{l}\text { SCN4A } \\
C A C N A 1 S \\
\text { KCNJ2 }\end{array}$ & $\begin{array}{l}\alpha \text { subunit, Nav1.4 skeletal muscle Na channel } \\
\alpha \text { subunit, Cav1.1, L-type Ca channel } \\
\text { Kir2.1 K channel inward rectifier }\end{array}$ \\
\hline Myotonia & $\begin{array}{l}S C N 4 A \\
C l C 1\end{array}$ & $\begin{array}{l}\alpha \text { subunit, Nav1.4 skeletal muscle } \mathrm{Na} \text { channel } \\
\text { ClC- } 1 \text { chloride channel }\end{array}$ \\
\hline Congenital Myasthenic Syndrome & $\begin{array}{l}\text { CHRNA1 } \\
\text { CHRNB1 } \\
\text { CHRNE } \\
\text { CHRND }\end{array}$ & $\begin{array}{l}\alpha \text { subunit, nicotinic acetylcholine receptor } \\
\beta \text { subunit, nicotinic acetylcholine receptor } \\
\epsilon \text { subunit, nicotinic acetylcholine receptor } \\
\delta \text { subunit, nicotinic acetylcholine receptor }\end{array}$ \\
\hline Malignant Hyperthermia & $\begin{array}{l}\text { CACNAIS } \\
\text { RYRl }\end{array}$ & $\begin{array}{l}\alpha \text { Subunit, Cav1.1 L-type Ca channel } \\
\text { Ca release channel / ryanodine receptor }\end{array}$ \\
\hline Central Core Disease & $R Y R l$ & Ca release channel / ryanodine receptor \\
\hline
\end{tabular}

References to the original reports of these mutations are available in several comprehensive reviews. ${ }^{3,4,5}$

bodies to the nicotinic acetylcholine receptor, which causes the profound fatigue and weakness of myasthenia gravis due to impairment in the safety factor of neuromuscular transmission from receptor block and internalization.

The notion that human disorders may arise from a heritable defect in an ion channel gene is a more recent concept. These ion channelopathies were first identified in disorders of skeletal muscle excitability (myotonia and periodic paralysis), ${ }^{2}$ and in the past 20 years the list has rapidly expanded to include more than 40 human diseases. The series of articles in this issue of Neurotherapeutics focuses on disorders of brain, peripheral nervous system, and skeletal muscle caused by mutations affecting the coding region of voltage-gated and fast ligandgated (ionotropic) ion channels (Table 1). Disease-associated mutations have been identified for all the major classes of voltage-gated ion channels $\left(\mathrm{Na}^{+}, \mathrm{K}^{+}, \mathrm{Ca}^{2+}\right.$, 
$\mathrm{Cl}^{-}$), for several fast ligand-gated receptors or channels (nicotinic acetylcholine receptor, glycine receptor, GABA receptor), for intracellular channels (ryanodine receptor, $\mathrm{Ca}^{2+}$ release channel), and for intercellular channels (connexins). These have been summarized in several reviews. ${ }^{3-5}$

The list of disorders in Table 1 illustrates three common features of ion channelopathies affecting brain and skeletal muscle. 1) Symptoms often present as paroxysmal attacks, with normal function interictally (e.g., epilepsy or periodic paralysis). Consistent with this observation, many channel mutations produce only modest functional changes or affect channels with redundant expression of functionally similar channels. 2) Most channelopathies are inherited as autosomal dominant traits. In part, this may be due to an ascertainment bias, because the genetic linkage and positional cloning techniques used to identify many channelopathies are more readily accomplished in kindreds with Mendelian autosomal dominant inheritance. 3) Most channelopathies cause single-organ involvement. For example, periodic paralysis disrupts skeletal muscle excitability but does not cause CNS symptoms, because the affected ion channel genes are expressed in skeletal muscle but not brain.

\section{CLASSES OF ION CHANNELS AND MOLECULAR STRUCTURE}

Historically, ion channels were classified and named on the basis of their physiological and pharmacological properties. For example, the voltage-gated $\mathrm{K}^{+}$channels are activated by membrane depolarization and selectively conduct $\mathrm{K}^{+}$ions. This scheme has been modified by the discovery of large gene families coding for functionally related ion channels. Many more channel genes were cloned than had been expected from the electrophysiological classification of channel types. Moreover, these genetic data revealed evolutionary patterns of channel diversification and inter-relatedness of channels with functionally divergent properties (e.g., voltagegated $\mathrm{K}^{+}$channels and cyclic nucleotide-gated channels). The channel classification scheme continues to undergo revision, but has retained many features of the historical system in which channels are classified by the stimuli to which they respond: voltage-gated, ligandgated, second messenger-gated, or special sensory stimuli (gated by stretch, $\mathrm{pH}$, or heat).

\section{Voltage-gated ion channels}

Voltage-gated ion channels are members of a large, diverse gene superfamily. ${ }^{6}$ Most ion channels in this family open in response to membrane depolarization and have a steep voltage dependence, for which the open probability $\left(P_{\text {open }}\right)$ increases 10 -fold for every 7 to $12 \mathrm{mV}$ of depolarization. Ion channels in this class in- clude the voltage-gated $\mathrm{Na}^{+}$and $\mathrm{K}^{+}$channels that are responsible for the generation and propagation of action potentials in neurons, skeletal muscle, and heart. The voltage sensitivity of these channels is derived from a transmembrane $\alpha$-helical segment that contains a cluster of four to eight positively charged amino acids (lysine or arginine). The charged residues form a dipole that moves outward in response to membrane depolarization ${ }^{7,8}$ and thereby influences the probability of adopting the closed or open conformation. This cluster of positive charges is in the so-called S4-segment, the fourth of six membranespanning segments in the canonical architecture of a channel subunit in the voltage-gated superfamily (FIG. 1). The presence of this S4 motif is a defining characteristic for members of the voltage-gated ion channel superfamily.

The prototypical structure for a voltage-gated $\mathrm{K}^{+}$ channel is illustrated by the model of a Shaker-type $\mathrm{K}$ channel (FIG. 1). Members of this Kv family are tetramers of $\alpha$-subunits, each comprised of approximately 800 amino acids with six hydrophobic stretches of residues that form the membrane-spanning segments (S1S6). The $\alpha$-subunits are assembled symmetrically around a central ion-conducting pore. The ionic selectivity of the pore is determined by a hydrophilic loop of 20 residues between the S5 and S6 segments. This pore loop is conserved among all $\mathrm{K}^{+}$-selective ion channels and contains the eight-residue sequence [T/S]-[M/L/Q]-T-T[I/V]-G-Y-G. This signature sequence has been used to identify a large superfamily of $\mathrm{K}$ channel genes in genomic and expressed sequence tag databases. The $\mathrm{X}$-ray crystal structure of a minimalist $\mathrm{K}$ channel from bacteria with only two membrane-spanning segments (homologs of S5, S6 in Shaker) revealed how the exquisite selectivity of $\mathrm{K}$ channels arises from interaction of the oxygen atoms along the main polypeptide chain (not the side groups that define specific amino acids) in the poreloop residues, which are precisely positioned to substitute for the oxygen atoms of adjacent water molecules that hydrate $\mathrm{K}^{+}$ions in free solution. ${ }^{9}$

Outward motion of the S4 segment is coupled to channel opening, probably by splaying apart the C-terminal ends of the S6 segments, which are believed to form the activation gate. ${ }^{10}$ In the presence of maintained depolarization, activated Shaker K channels shut within a few milliseconds to a nonconducting inactivated state. This $\mathrm{N}$-type inactivation occurs by occlusion of the pore by a tethered blocker formed by the intracellular amino terminus of the $\alpha$-subunit. ${ }^{11}$ Shaker K channels may also inactivate by alternative mechanisms. Collapse of the permeation pathway at the outer mouth of the pore by motion of the S6 segment gives rise to C-type inactivation. ${ }^{12}$ Voltage-gated $\mathrm{Na}^{+}$channels (Nav) and $\mathrm{Ca}^{2+}$ channels (Cav) share sequence similarity and conserved structural features with Shaker $\mathrm{K}^{+}$channels, except that 

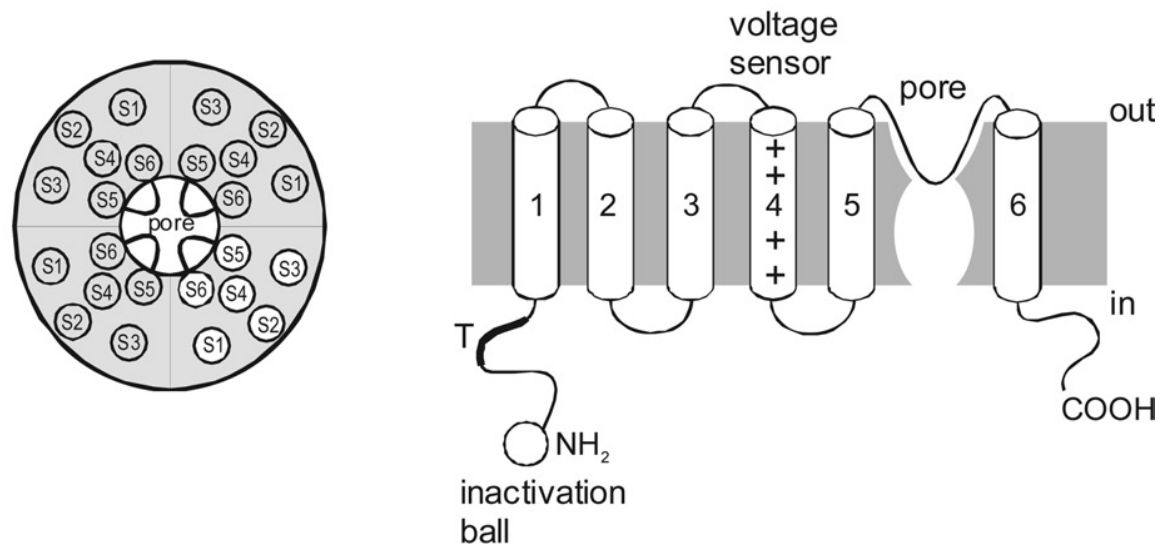

B
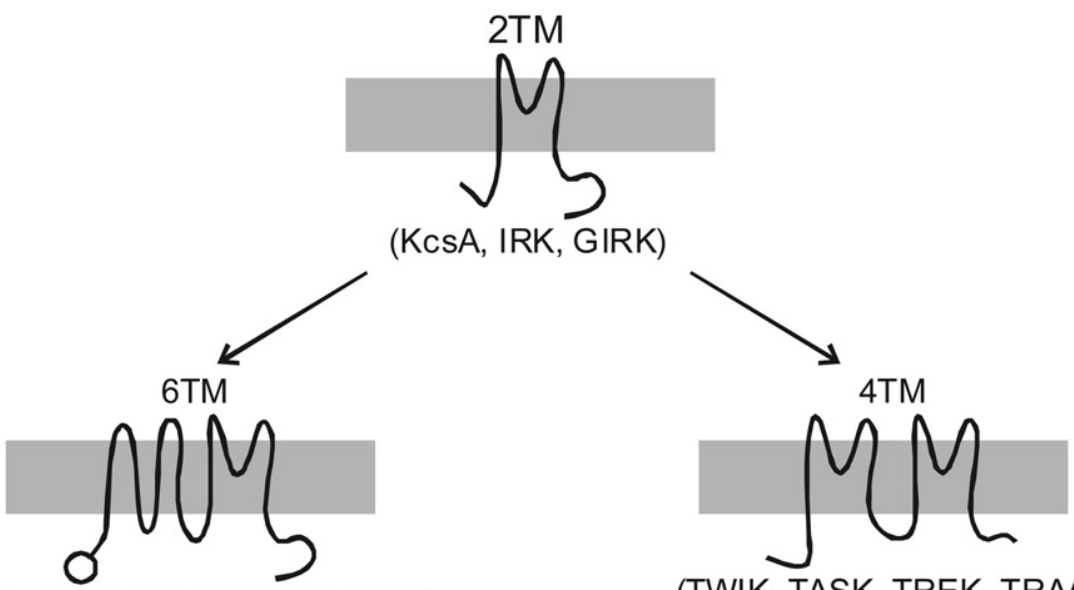

(Kv, KQT, BK, SK, EAG, CNG)

(TWIK, TASK, TREK, TRAAK)

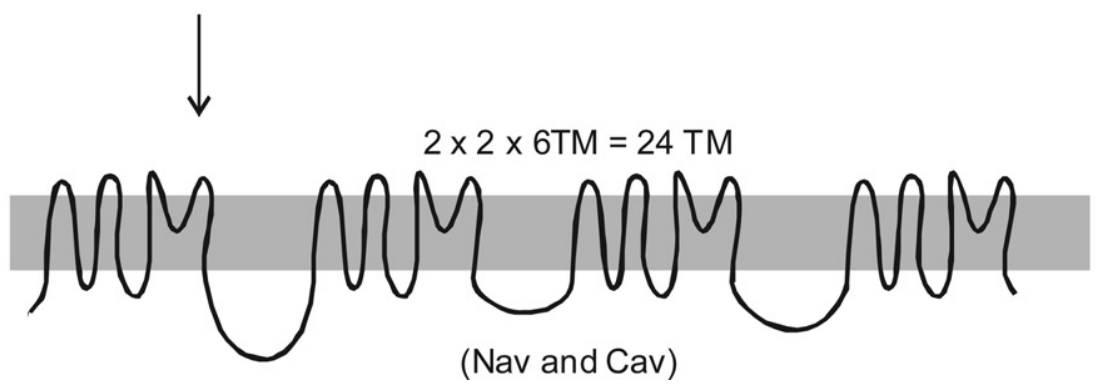

FIG. 1. Membrane topology and functional domains of voltage-gated ion channels. (A) Top view looking down on the plane of the membrane (left) shows the fourfold symmetry of subunits (Kv channels) or homologous repeats (Nav or Cav channels) around a central pore. Each subunit contains six transmembrane segments (S), shown opened into a linear membrane-folding diagram (right). The loop between S5 and S6 forms the pore, and the voltage sensor is denoted by the positive charges in S4. For Kv channels, the cytoplasmic amino terminus acts as an inactivation ball that plugs the pore in open channels, and the tetramerization domain $(T)$ regulates which $\alpha$-subunits can assemble to form homo- or heteromeric channels. (B) Evolution of the voltage-gated ion channel superfamily is believed to have resulted from duplications of a primordial two transmembrane (TM) $\mathrm{K}^{+}$channel gene. The 2TM group includes bacterial (KcsA) and inward rectifier $\mathrm{K}^{+}$channels (IRK, and G-protein coupled GIRK). A single duplication produced the 4TM group, with two pore regions in each $\alpha$-subunit. These channels (TWIK, TASK, TREK, TRAAK) act as leakage $\mathrm{K}^{+}$-selective channels that are open at all membrane voltages and are thought to play a major role is setting the resting potential. Strong voltage sensitivity first appears in the 6TM group, which includes voltage-gated (Kv, KQT, EAG), $\mathrm{Ca}^{2+}$-activated (BK, SK), and cyclic nucleotide-gated (CNG) channels. CNG channels are nonselective cation channels; all other members of the 6TM group are highly $\mathrm{K}^{+}$selective. Two rounds of gene duplication gave rise to the 24TM group, which includes voltage-gated Nav and Cav channels. Each of the four internal repeats is homologous to a 6TM subunit of $\mathrm{Kv}$ channels, and a single $\alpha$-subunit is capable of forming a functional channel. Modified from Cannon, 2001.25 
they contain a single pore-forming $\alpha$-subunit homologous to four concatenated Shaker $\alpha$-subunits. This sequence conservation suggests that Nav and Cav channels evolved from two rounds of duplication of a primordial $\mathrm{Kv}$-like gene. The cytoplasmic loop between the third and fourth homologous domains in Nav channels forms the fast-inactivation gate. ${ }^{13}$

Some ion channels containing an S4 voltage-gating motif are relatively insensitive to membrane potential. $\mathrm{Ca}^{2+}$-activated $\mathrm{K}$ channels are activated by internal $\mathrm{Ca}^{2+}$ in the micromolar range, but have much lower voltage sensitivity than other S4-containing K channels. Cyclic nucleotide-gated channels in retina and olfactory epithelia are potently modulated by cytoplasmic cGMP and cAMP levels, respectively, but also contain an S4 domain and can be activated by depolarization.

Other subfamilies of K-selective channels lack intrinsic voltage sensitivity. The inward-rectifying $\mathrm{K}$ channels (Kir) are so named because they allow inward movement of $\mathrm{K}^{+}$at negative voltages much more readily than outward movement of $\mathrm{K}^{+}$at positive voltages. This rectification (as in a diode that passes electrical current in one direction) occurs because intracellular cations $\left(\mathrm{Mg}^{2+}\right.$ or organic polyamines) create a strong voltage-dependent block of Kir channels that is favored by depolarization. ${ }^{14}$ Subunits of Kir channels contain only two transmembrane segments, homologous to S5-S6 of the Shaker K channel and the intervening pore loop. Another class of voltage-independent $\mathrm{K}$ channel has two pore loops within a single subunit, each of which has four transmembrane segments. Several subfamilies of these twopore $\mathrm{K}$ channels have been identified by searching databases for the tandem K-selectivity motif. ${ }^{15}$ These channels (Task, Twik, Trek, Traak) are constitutively open, and their activity is modulated by $\mathrm{pH}$, phosphorylation, temperature, and stretch. Because Kir and twopore $\mathrm{K}$ channels are open at negative voltages, they are believed to be important determinants in setting the resting membrane potential.

Not all voltage-gated ion channels contain an S4 motif. Chloride channels in the $\mathrm{ClC}$ family are homodimers in which each subunit contains an anion-selective pore, but no S4 sequence. This double-barreled channel is activated by depolarization, which increases the $P_{\text {open }}$ for each pore individually and activates a common gate that regulates accessibility of both pores simultaneously. ${ }^{16}$

In addition to the main pore-forming $\alpha$-subunits, voltage-gated ion channel complexes contain associated auxiliary subunits. ${ }^{17}$ These auxiliary subunits may be intracellular, membrane-spanning, or extracellular and bind either noncovalently or by covalent disulfide linkage to $\alpha$-subunits. By definition, auxiliary subunits cannot form ion-conducting channels, but when coexpressed with $\alpha$-subunits they modify channel gating, expression level at the membrane, targeting to subcellular domains, pharmacologic properties, and even selectivity. Mutations in auxiliary subunit genes have been identified as a rare cause of epilepsy, ${ }^{18}$ but the overwhelming majority of channelopathies are associated with mutations in the pore-forming $\alpha$-subunit.

\section{Fast ligand-gated channels}

Ligand-gated channels at fast synapses contain highaffinity binding sites that, when occupied, are allosterically coupled to activation of an ion-conducting pore in the same molecular complex. Most of these channels are heteropentamers assembled around a central pore: nicotinic acetylcholine receptor, nAChR; glycine receptor, GlyR; $\gamma$-aminobutyric acid receptors type-A receptor, $\mathrm{GABA}_{\mathrm{A}} \mathrm{R}$; 5-hydroxytryptamine (serotonin) receptor type 3A, 5- $\mathrm{HT}_{3 \mathrm{~A}}$. Each subunit contains four transmembrane segments (M1-M4). The amino terminus is extracellular and forms the ligand binding site. Residues in the M2 segment line the pore and determine ion selectivity. Three conserved rings of negatively charged amino acids lining the pore result in the cation selectivity of nAChRs and $5-\mathrm{HT}_{3 \mathrm{~A}}$ receptors at excitatory synapses.

In contrast to voltage-gated cation channels, which are highly selective for a specific cation (e.g., $\mathrm{K}^{+}$), these excitatory ligand-gated receptors have nonselective cation channels that do not discriminate between $\mathrm{Na}^{+}$or $\mathrm{K}^{+}$, and even have an appreciable permeability to $\mathrm{Ca}^{2+}$. The inhibitory receptors $\left(\mathrm{GlyR}, \mathrm{GABA}_{\mathrm{A}} \mathrm{R}\right)$ are anion permeant $\left(\mathrm{Cl}^{-}\right.$being the most important biologically), because the middle ring of charge is replaced by a neutral alanine plus a helix-deforming proline and the outer ring contains positively charged residues. Glutamate receptors (GluR) mediate excitatory synaptic transmission and have a different topology. Both the AMPA and NMDA subtypes of GluR are tetramers. Another difference is that the M2 segment makes a hairpin turn and does not completely cross the membrane. As a result, the M3-M4 loop is extracellular and the carboxyl terminus is intracellular.

\section{ASSESSMENT OF ION CHANNEL FUNCTION}

In the context of exploring the pathogenesis of ion channelopathies, the functional consequences of diseaseassociated mutations have been characterized most extensively in terms of direct effects on channel gating or permeation. This approach has been readily achieved by using the same exogenous expression systems that were developed to characterize the normal function of cloned channels. Expression in frog oocytes or cell lines (e.g., human embryonic kidney cells) offers the advantage of studying the behavior a relatively pure population of channels expressed at very high levels, compared to the endogenous background channels. Potential limitations include the absence of interacting accessory proteins, an 
unnatural cellular milieu for which channel modulation or regulation may be altered, and difficulties interpreting the significance of expression level, protein turnover, and protein trafficking to subcellular compartments. Nevertheless, a remarkable degree of insight on disease pathogenesis has been achieved by studying the functional properties of mutant channels expressed in artificial systems. These cell-based systems are now being extended to whole-organism studies in genetically engineered mice, worms, flies, or zebrafish.

The functional properties of channels under study are characterized by recording ionic current that flows under controlled conditions of membrane potential, ligand, or other stimulus. Current may be measured as the summed activity from all of the active channels in the entire cell (whole-cell mode) or as the individual currents through a single channel or a small number of channels in an isolated patch of membrane (patch-clamp mode). The current is a measure of the net movement of ions across the membrane per unit time and is a manifestation of both intrinsic channel properties and of the experimental conditions. For example, the amplitude of the current will depend on intrinsic properties of the channel: the probability of the channel being open (i.e., gating) and how well each type of ion passes through the pore (i.e., permeation) as well as extrinsic features of the experiment: the membrane potential, $V_{\mathrm{m}}$, and the equilibrium potential for each permeant ion.

The equilibrium potential is the membrane voltage at which net movement of a specific ion (e.g., $\mathrm{K}^{+}$) would be zero because the tendency for efflux exactly balances influx. The equilibrium potential (or Nernst potential) is a consequence of the concentration gradient of the ion across the membrane and is not a feature of the channel per se. This equilibrium value is proportional to the logarithm of the concentration ratio $[\text { ion }]_{\text {in }} /[\text { ion }]_{\text {out }}$. The difference between the membrane potential, $V_{\mathrm{m}}$, which is typically controlled experimentally, and the equilibrium potential, $E_{\mathrm{ion}}$, is often referred to as the driving force on the ion.

Ion channels are not perfectly selective in allowing one type of ion to pass through to pore and excluding all others. Voltage-gated ion channels have relatively high selectivity, so for example a K-channel conducts $1 \mathrm{Na}^{+}$ ion for every $100 \mathrm{~K}^{+}$ions in a competition experiment with $\mathrm{Na}^{+}$being the only cation on one side of the membrane and $\mathrm{K}^{+}$on the other side. Conversely, ligand-gated cation channels are nonselective, so that $\mathrm{Na}^{+}$and $\mathrm{K}^{+}$ pass equally well through the $\mathrm{nAChR}$ at the muscle endplate. Because ion channels are not perfectly selective and there are many types of permeant ions present under physiological conditions, the voltage at which the net current flow through the channel is zero will not be the equilibrium potential for a specific ion.

The voltage at which the net current flow through a channel is zero is called the reversal potential, $E_{\mathrm{rev}}$, because it is the voltage at which the current reverses direction: $V_{\mathrm{m}}>E_{\text {rev }}$ produces an outward current flow, whereas $V_{\mathrm{m}}<E_{\text {rev }}$ results in net inward current. The reversal potential depends on both the intrinsic selectivity properties of the channel and the concentration of ions on either side of the membrane. If a channel were perfectly selective, to $\mathrm{K}^{+}$for example, then $E_{\text {rev }}$ would equal the Nernst potential for $\mathrm{K}^{+}, E_{\mathrm{K}}$. In actuality, however, $\mathrm{K}^{+}$channels are also permeable to $\mathrm{Na}^{+}$, so $E_{\mathrm{rev}}$ for a $\mathrm{K}$ channel has an intermediate value between $E_{\mathrm{K}}$ and $E_{\mathrm{Na}}$. In this case, $E_{\mathrm{rev}}$ is much closer to $E_{\mathrm{K}}$ than $E_{\mathrm{Na}}$ because the channel is more permeable to $\mathrm{K}^{+}$than $\mathrm{Na}^{+}$.

The reversal potential of a channel is an important concept for understanding how the activity of a channel affects the membrane potential. Net current will flow through an open channel whenever $V_{\mathrm{m}}$ does not equal $E_{\mathrm{rev}}$, and the effect of this current is to shift $V_{\mathrm{m}}$ toward $E_{\mathrm{rev}}$. In actual cells, many types of channels are present, each with its own $E_{\mathrm{rev}}$. The membrane potential will be a weighted average of the reversal potentials for all these channel types, with the weight being proportional to the number of channels and the probability of being open. Stated another way, the effect of channel opening on $V_{\mathrm{m}}$ is to move the membrane potential closer to the reversal potential for that channel.

Under physiological conditions, the current flow $(I)$ through a channel is proportional to the driving force, $V_{\mathrm{m}}-E_{\text {rev: }}$ :

$$
I=G \times\left(V_{\mathrm{m}}-E_{\mathrm{rev}}\right) .
$$

The proportionality constant, $G$, is the conductance, which is the reciprocal of resistance in the familiar Ohm's law: voltage equals current times resistance. Notice that if $V_{\mathrm{m}}$ equals $E_{\mathrm{rev}}$, there is no driving force and the current will be zero, even if $100 \%$ of the channels are open. The intrinsic features of the channel are all contained in the conductance term, $G$. The interpretation of $G$ in relation to the properties of an individual ion channel can be understood by considering a simple mass balance. The total current $(I)$ must equal the amount of current carried by a single channel (i) times the number of channels in the membrane $(N)$, times the probability that any given channel is open $\left(P_{\text {open }}\right)$ :

$$
I=N \times P_{\text {open }} \times i .
$$

The current through an individual channel is proportional to the driving force on the ion (voltage), analogous to the macroscopic relation in equation (1), but now $\gamma$ is the conductance for an individual channel:

$$
i=\gamma \times\left(V_{\mathrm{m}}-E_{\mathrm{rev}}\right) .
$$

Combining equations (2) and (3) yields 


$$
I=N \times P_{\text {open }} \times \gamma \times\left(V_{\mathrm{m}}-E_{\text {rev }}\right) .
$$

Equation (4) illustrates how intrinsic properties of an ion channel $\left(N, P_{\text {open }}\right.$, or $\gamma$, any of which might be altered by a disease-associated mutation) and extrinsic features of the experimental paradigm $\left(V_{\mathrm{m}}, E_{\mathrm{rev}}\right)$ affect the current measured experimentally, $I$. Therefore, in the analysis of ion channel function, the measured current is normalized by the driving force $\left(V_{\mathrm{m}}-E_{\mathrm{rev}}\right)$ to ascertain the intrinsic properties of the channel:

$$
G=N \times P_{\text {open }} \times \gamma=I /\left(V_{\mathrm{m}}-E_{\text {rev }}\right) .
$$

The conductance term summarizes both the gating properties of the channel, $P_{\text {open }}$, and the permeability of a specific ion, $\gamma$. Over the time course of an experiment, $N$ and $\gamma$ remain constant, and the time-dependent change of the measured current amplitude in response to a step change in voltage (or other stimulus, such as ligand concentration, $\mathrm{pH}$, stretch, temperature) is attributed to the kinetics of the gating process as channels shift closed to open or vice versa. Thus, for voltage-gated channels the open probability is a function of voltage and time, $P_{\text {open }}\left(V_{\mathrm{m}}, t\right)$.

In practice, the assessment of ion channel function is performed on channels artificially expressed in a model system for which current is measured in response to manipulation of membrane potential, ligand concentration, or other form of stimulus. Permeation is characterized by measuring the reversal potential (experimentally adjusting $V_{\mathrm{m}}$ until the current is zero). A more detailed analysis may include measuring the change in $E_{\text {rev }}$ produced by replacing the extracellular saline with various test ions, from which the relative permeability of these ions can be quantified. If currents are measured through individual channels by patch-clamp recording, then the unitary conductance, $\gamma$, can be determined as the ratio $i /\left(V_{\mathrm{m}}-E_{\text {rev }}\right)$ as another indicator of permeation.

Gating is characterized by measuring the change in conductance as the channel stimulus is varied. For voltage-gated channels, the current is measured at a series of test potentials applied as voltage steps and normalized by the driving force $\left(V_{\mathrm{m}}-E_{\text {rev }}\right)$ to compute conductance. These data could be a measure of peak conductance for a channel that opens transiently (e.g., $\mathrm{Na}^{+}$channel) or steady-state conductance for a channel that remains open persistently. The conductance-voltage relation, or $G\left(V_{\mathrm{m}}\right)$ curve, is a measure of channel voltage-dependent activation. For steps from negative potentials where channels are closed (e.g., $-100 \mathrm{mV}$ ) to more positive potentials where channels open, the time course for the increase in conductance provides a measure of the kinetics of activation. Alternatively, the voltage step can begin from a depolarized value and jump to a more negative potential that closes the channel, from which the decay in current reflects the kinetics of deactivation.
After opening, most channels eventually stop conducting current even in the continued presence of the stimulus. Increasing the intensity of the stimulus (e.g., further depolarization) does not cause channels to open. This behavior is termed inactivation and is different from the closed or resting state of the channel because inactivated channels are refractory from being opened by the application of a stimulus. For ligand-gated channels, this process is termed desensitization. Channels recover from inactivation by resetting the membrane potential to a negative voltage (or washing out the ligand) that favors conversion back to the closed conformation of the channel. Inactivation is quantified by determining the voltage dependence for shifting channels to the inactive state (measured as the proportional reduction in peak current caused by a depolarized shift in the starting potential from which the test depolarization is applied), as well as the time course for entering into or recovering from inactivation.

\section{ION CHANNEL DEFECTS IN CHANNELOPATHIES OF BRAIN AND MUSCLE}

Functional assessment of ion channel mutations associated with human disorders of brain and skeletal muscle has revealed a wide variety of pathogenic changes. In most cases, these studies have been performed in artificial expression systems wherein a cDNA coding for the mutant channel is transfected into a fibroblast or mRNA is injected into an oocyte. Ionic current is then measured under voltage-clamp conditions to characterize the functional behavior of the expressed channels. In many cases, these mutant proteins can assemble into functional channels that conduct ionic current but have altered gating properties. Gating defects in mutant channels may appear subtle under voltage-clamp, but the consequences under physiological conditions on the resting membrane potential, action potential threshold, pacemaker activity, or the fidelity of synaptic transmission can be dramatic.

Isolated defects in channel permeation or selectivity are observed much less frequently, either because mutations in the pore loop destroy the ability to conduct any current or possibly because a loss of selectivity is catastrophic for cell viability and is therefore embryonic lethal. The ability to compare the behavior of wild-type and mutant ion channels with such high precision, coupled with the known role of most ion channels in regulating electrical excitability, has provided insights on the pathomechanism of the channelopathies that have not yet been possible for most neurogenetic disorders.

Many channelopathies with a dominant inheritance pattern are caused by gain-of-function defects in which the open probability of mutant channels is greater than normal. This type of defect is particularly common for 


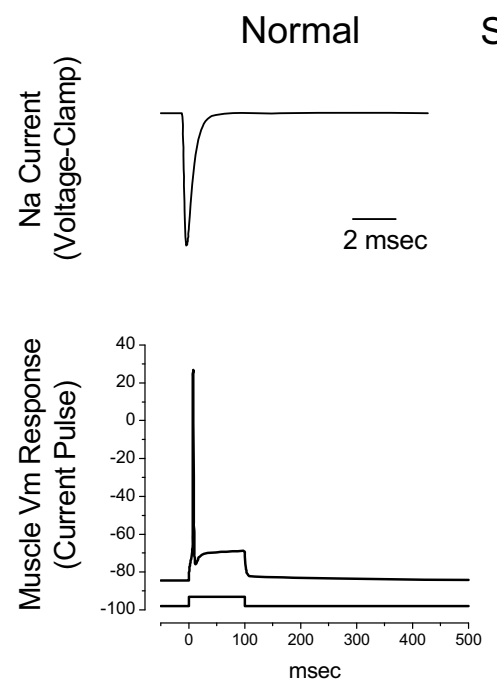

Slowed Inactivation

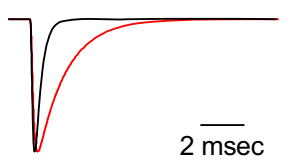

myotonia

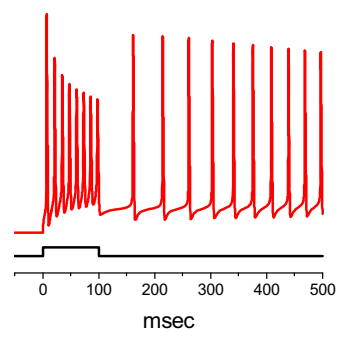

Incomplete Inactivation

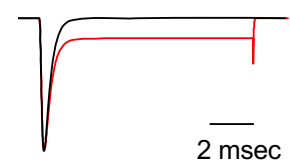

periodic paralysis

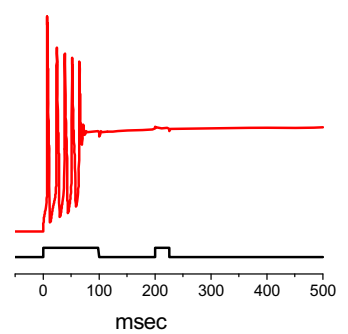

FIG. 2. Gain-of-function changes for skeletal muscle $\mathrm{Na}^{+}$channel mutations in myotonia and periodic paralysis. The top row shows representative $\mathrm{Na}^{+}$currents recorded in response to a step depolarization from $-100 \mathrm{mV}$ to $-10 \mathrm{mV}$ for normal channels (left) or gain-of-function defects caused by a slower rate of inactivation (center) or incomplete inactivation (right). The consequences of these alterations in $\mathrm{Na}^{+}$channel gating are shown for the computer simulations of muscle fiber $V_{m}$ in the bottom row (note change in time scale relative to top row). Normally. only a single action potential is elicited by a suprathreshold current pulse (left). A fivefold slowing of $\mathrm{Na}^{+}$ channel inactivation produces a dynamic instability with increased susceptibility to after-discharges, which produce the persistent contraction of myotonic stiffness (center). A different type of inactivation defect in which a persistent Na current is conducted due to incomplete inactivation (right) produces a stable depolarization of $V_{\mathrm{m}}$, which renders the fiber refractory due to the normal voltagedependent inactivation of $\mathrm{Na}^{+}$channels.

$\mathrm{Na}^{+}$channel mutations found in periodic paralysis and myotonia of skeletal muscle ${ }^{4}$ or for some forms of epilepsy. ${ }^{19}$ The gain of function may be due to impaired inactivation wherein mutant channels inactivate abnormally slowly or incompletely (FIG. 2). Alternatively, the gain of function may be due to enhanced activation in which mutant channels open more readily because the voltage dependence of channel activation is shifted left toward more hyperpolarized potentials. The $\mathrm{Na}^{+}$current depolarizes the cell, and as expected, gain of function defects for many $\mathrm{Na}^{+}$channelopathies lead to enhanced excitability with increased firing rates or bursts of autonomous discharges, as occurs in some epilepsies or in myotonia. An aberrantly persistent $\mathrm{Na}^{+}$current may also cause a loss of excitability. In hyperkalemic periodic paralysis, for example, impairment of inactivation leads to a small fraction of persistently open mutant channels $(\sim 3 \%)$. The steady inward $\mathrm{Na}^{+}$may sufficiently depolarize the resting potential to produce flaccid electrically inexcitable muscle by voltage-dependent inactivation of the normally behaving $\mathrm{Na}^{+}$channels (both wild-type and most of the mutant ones). This example illustrates that a gain-of-function defect at the channel biophysical level may produce either a gain or loss of excitability at the cellular level.

Channel mutations may also cause loss-of-function defects giving rise to recessively or dominantly inherited clinical phenotypes. The mutant allele may have lost the ability to encode a functional channel subunit due to a nonsense mutation with a premature termination codon or an RNA splice donor-acceptor defect. This type of defect may produce an asymptomatic carrier in the heterozygous state, as occurs, for example, with $\mathrm{ClC}-1 \mathrm{mu}-$ tations in recessive myotonia congenita. The chloride conductance must be reduced to less than $50 \%$ of normal before the muscle will develop susceptibility to myotonic discharges, and so only homozygotes or compound heterozygotes carrying two mutant alleles are symptomatic.

In other disorders, a loss of one normal allele or haploinsufficiency is not tolerated. Severe myoclonic epilepsy of infancy occurs in heterozygotes with a nonsense mutation in the Nav1.1 neuronal sodium channel. ${ }^{20} \mathrm{~A}$ loss-of-function mutation may also result from a dominant-negative effect on the multimeric composition of the channel complex. For example, missense mutations of the Kir2.1 K channel in Anderson-Tawil syndrome

TABLE 2. Variables Defined

\begin{tabular}{ll}
\hline Variable & \multicolumn{1}{c}{ Definition } \\
\hline$E_{\mathrm{K}}$ & $\begin{array}{c}\text { Nernst or equilibrium potential for } \mathrm{K} \\
\text { (potassium) } \\
E_{\mathrm{rev}}\end{array}$ \\
$\begin{array}{l}\text { reversal potential } \\
\text { conductance for the entire cell: } G=N \times \\
P_{\text {open }} \times \gamma\end{array}$ \\
$\gamma$ & $\begin{array}{l}\text { conductance for an individual channel } \\
\text { ionic current through an individual channel }\end{array}$ \\
$I$ & ionic current passing through the entire cell \\
$P_{\text {open }}$ & probability that a channel is open \\
$V_{\mathrm{m}}$ & membrane potential \\
\hline
\end{tabular}


not only fail to form functional (homomeric) channels, they also combine with wild-type subunits and interfere with channel function. ${ }^{21}$ Loss-of-function defects may alter channel function without destroying the capability of forming an ion-conducting pore. Missense mutations in $\mathrm{ClC}-1$ that reduce channel opening by shifting activation toward depolarized voltages in both homomeric mutant channels and heteromeric mutant-wild-type complexes cause dominantly inherited myotonia congenita. ${ }^{22}$

Alteration in permeability has been observed for a few channelopathies. Some missense mutations in the Cav2.1 $\mathrm{P} / \mathrm{Q}$-type $\mathrm{Ca}^{2+}$ channel that occur in familial hemiplegic migraine have a reduced unitary conductance. ${ }^{23}$ Autosomal dominant nocturnal frontal lobe epilepsy results from a serine to phenylalanine mutation in the pore region (M2) of the $\alpha_{4}$ nicotinic acetylcholine receptor subunit that alters both permeation and gating. In mutant channels, the single-channel conductance is reduced nearly twofold, permeability to $\mathrm{Ca}^{2+}$ is lost, desensitization occurs more rapidly, and recovery from desensitization is prolonged. ${ }^{24}$

Several common themes have emerged from functional studies on ion channel mutations found in channelopathies. First, most defects tend to produce membrane depolarization; loss-of-function changes are commonly observed for $\mathrm{K}^{+}$channel and $\mathrm{Cl}^{-}$channel mutations, whereas $\mathrm{Na}^{+}$ channel mutations often cause gain-of-function changes. Second, mutants that express and form functional channels more commonly affect gating than permeation. Third, the functional changes in mutant channel behavior are often subtle, which likely contributes to the paroxysmal nature of the symptoms in these disorders. The susceptibility for disruption of cellular excitability is increased by these mild changes, but an environmental trigger such as temperature change or fluctuation in ion concentration is required to manifest clinical symptoms.

Recent progress in elucidating the pathomechanisms of ion channelopathies has been a remarkable achievement and provides one of the best examples for tracing the basis of a human disorder from gene defect to changes in protein function to altered cellular excitability and finally to clinical symptoms. Several factors contributed to the rapid progress in this field. Well-defined kindreds with clearly identified affected individuals and dominant inheritance patterns aided in the identification of disease genes through genetic linkage and positional cloning. More importantly, the functional properties and physiological role of ion channels were already well established. The highly sensitive methods for characterizing channel function with voltage-clamp techniques were critical to identifying the rather subtle changes caused by many mutations. Finally, the consequences of specific channel defects could be linked mechanistically to alterations in cellular excitability that give rise to the clinical phenotype.
The next major advances in the field of channelopathies will likely come from the study of genetically engineered mice that recapitulate human disorders. These models will provide a more accurate picture for the alterations in channel function in their native cellular milieu and will provide an opportunity to systematically investigate the mechanistic link between environmental triggers and transient alterations in cellular excitability that produce symptoms. A greater understanding of the origins for the paroxysmal dysfunction of brain or skeletal muscle in these disorders will provide important insights for opportunities to intervene therapeutically.

Acknowledgments: Work in the author's laboratory has been supported by the National Institute of Arthritis and Musculoskeletal and Skin Diseases and the National Institute of Neurological Disorders and Stroke of the U.S. National Institutes of Health; the Muscular Dystrophy Association; and the Foundation for Anesthesia Education and Research.

\section{REFERENCES}

1. Hille B. Ion channels of excitable membranes, 3rd ed. Sunderland, MA: Sinauer, 2001.

2. Adrian RH, Bryant SH. On the repetitive discharge in myotonic muscle fibres. J Physiol 1974;240:505-515.

3. Ashcroft FM. Ion channels and disease. San Diego: Academic Press, 2000.

4. Cannon SC. Pathomechanisms in channelopathies of skeletal muscle and brain. Annu Rev Neurosci 2006;29:387-415.

5. Lehmann-Horn F, Jurkat-Rott K. Voltage-gated ion channels and hereditary disease. Physiol Rev 1999;79:1317-1372.

6. Catterall WA. Structure and function of voltage-gated ion channels. Annu Rev Biochem 1995;64:493-531.

7. Mannuzzu LM, Moronne MM, Isacoff EY. Direct physical measure of conformational rearrangement underlying potassium channel gating. Science 1996;271:213-216.

8. Yang N, George AL Jr, Horn R. Molecular basis of charge movement in voltage-gated sodium channels. Neuron 1996;16:113-122.

9. Doyle DA, Morais Cabral J, Pfuetzner RA, et al. The structure of the potassium channel: molecular basis of $\mathrm{K}^{+}$conduction and selectivity. Science 1998;280:69-77.

10. Yellen G. The moving parts of voltage-gated ion channels. Q Rev Biophys 1998;31:239-295.

11. Hoshi T, Zagotta WN, Aldrich RW. Biophysical and molecular mechanisms of Shaker potassium channel inactivation. Science 1990;250:533-538

12. Liu Y, Jurman ME, Yellen G. Dynamic rearrangement of the outer mouth of a K ${ }^{+}$channel during gating. Neuron 1996;16:859-867.

13. Patton DE, West JW, Catterall WA, Goldin AL. Amino acid residues required for fast $\mathrm{Na}^{+}$-channel inactivation: charge neutralizations and deletions in the III-IV linker. Proc Natl Acad Sci U S A 1992;89:10905-10909.

14. Matsuda H, Saigusa A, Irisawa H. Ohmic conducatance through the inwardly rectifying $\mathrm{K}$ channel and blocking by internal $\mathrm{Mg}^{2+}$. Nature 1987;325:156-159.

15. Ketchum KA, Joiner WJ, Sellers AJ, Kaczmarek LK, Goldstein SA. A new family of outwardly rectifying potassium channel proteins with two pore domains in tandem. Nature 1995;376:690695.

16. Jentsch TJ, Friedrich T, Schriever A, Yamada H. The CLC chloride channel family. Pflugers Arch 1999;437:783-795.

17. Isom LL, De Jongh KS, Catterall WA. Auxiliary subunits of voltage-gated ion channels. Neuron 1994;12:1183-1194.

18. Wallace RH, Wang DW, Singh R, et al. Febrile seizures and generalized epilepsy associated with a mutation in the $\mathrm{Na}^{+}$-channel $\beta 1$ subunit gene $S C N 1 B$. Nat Genet 1998;19:366-370. 
19. Rhodes TH, Lossin C, Vanoye CG, Wang DW, George AL Jr. Noninactivating voltage-gated sodium channels in severe myoclonic epilepsy of infancy. Proc Natl Acad Sci U S A 2004;101:11147-11152.

20. Sugawara T, Mazaki-Miyazaki E, Fukushima K, et al. Frequent mutations of SCN1A in severe myoclonic epilepsy in infancy. Neurology 2002;58:1122-1124.

21. Plaster NM, Tawil R, Tristani-Firouzi M, et al. Mutations in Kir2.1 cause the developmental and episodic electrical phenotypes of Andersen's syndrome. Cell 2001;105:511-519.

22. Fahlke C, Rüdel R, Mitrovic N, Zhou M, George AL Jr. An aspartic acid residue important for voltage-dependent gating of human muscle chloride channels. Neuron 1995;15:463-472.
23. Hans M, Luvisetto S, Williams ME, et al. Functional consequences of mutations in the human $\alpha 1 \mathrm{~A}$ calcium channel subunit linked to familial hemiplegic migraine. J Neurosci 1999; 19:1610-1619.

24. Kuryatov A, Gerzanich V, Nelson M, Olale F, Lindstrom J. Mutation causing autosomal dominant nocturnal frontal lobe epilepsy alters $\mathrm{Ca}^{2+}$ permeability, conductance, and gating of human $\alpha 4 \beta 2$ nicotinic acetylcholine receptors. J Neurosci 1997; 17:9035-9047.

25. Cannon S. Physiology of ion channels. In: Rose M, Griggs R, editors. Channelopathies of the nervous system. Oxford: Butterworth-Heinemann; 2001:2-15. 